\title{
ON-DEMAND RECEIVE FILTERING IN AN UMTS TERMINAL
}

\author{
J. Potman, R. Schiphorst and C.H. Slump \\ University of Twente \\ Department of Electrical Engineering, \\ Mathematics and Computer Science \\ P.O. Box 217 \\ NL-7500AE Enschede \\ The Netherlands \\ \{j.potman, r.schiphorst, c.h.slump\}@ewi.utwente.nl
}

\begin{abstract}
This paper describes a novel on-demand receive filter in an UMTS terminal. The receive filter is the first function after the Analog-to-Digital converter and is for that reason one of the most computation intensive parts in a receiver. The proposed filter architecture measures the out-of-band interference and calculates the required attenuation, which is used to select the appropriate filter. To assess the advantages of this on-demand receive filter, we have carried out field strength measurements in the UMTS FDD downlink band $(2.1 \mathrm{GHz})$. These measurements were carried out in Amsterdam, a dense urban area with 5 active UMTS operators. Our measurement results show that in a live network configuration there is almost no out-of-band interference. Moreover, an on-demand filter would save in this case more than $68 \%$ power compared to a fixed conventional receive filter.
\end{abstract}

\section{INTRODUCTION}

Since the introduction of second generation mobile communication systems such as GSM, mobile communication has become a major business. Moreover, due to Moore's law more and more parts of the receiver are being digitally implemented. Digitalization also enables the implementation of flexible receiver architectures. In this paper an example of adaptive signal processing is described. Nowadays, transceivers are designed for a worst case scenario that is defined in the standard. However, in most cases these scenarios do not apply and the overdesign results in extra hardware and power consumption. A better solution would be to measure the conditions under which the wanted signal is received and only apply the necessary signal processing for proper demodulation. This on-demand signal processing is called adaptive signal processing. Here, we describe an on-demand receive filter

This research is performed as part of the $\mathrm{AWgN}$ project which is supported by the Freeband Knowledge Impulse program, a joint initiative of the Dutch Ministry of Economic Affairs, knowledge institutions and industry. for UMTS (Universal Mobile Telecommunications System) FDD (Frequency Division Duplex). The receive filter provides the channel selection function and is the first function after the ADC (Analog-to-Digital Converter). For that reason, it operates at a high data rate and is one of the computational intensive parts in a receiver.

\subsection{Outline}

The outline of this paper is as follows. First, an introduction will be given on a flexible receive filter structure that can be used in an UMTS handset. On-demand filtering is only beneficial if worst case conditions rarely occur. To investigate this, we have measured the field strength in the UMTS downlink band $(2.1 \mathrm{GHz})$. The results of this campaign are described in a separate section. Finally, conclusions are drawn.

\section{AN ON-DEMAND RECEIVE FILTER}

\subsection{UMTS requirements}

Not much literature is known about on-demand receive filters for UMTS. A receive filter is used for selecting the appropriate channel in the UMTS frequency band. In [1] the worst case channel-selection selectivity requirements were determined from the UMTS receiver characteristics for adjacent channel selectivity, in- and out-of-band blocking and intermodulation presented in [2]. The filter has to provide a stopband attenuation of $33 \mathrm{~dB}$ at a frequency offset of $5 \mathrm{MHz}$ and a stop-band attenuation of $66 \mathrm{~dB}$ at frequency offsets of $10 \mathrm{MHz}$ and higher. The values are also called ACS (Adjacent Channel Selectivity) requirements.

However, ACI (Adjacent Channel Interference) consists of two parts: ACS and ACLR (Adjacent Channel Leakage Ratio). The latter defines the ratio between transmitted and out-of-band emissions in the base stations. If the desired signal has a lower energy than the leakage of the adjacent channel (ACLR), no reception is possible of the adjacent channel, 
even if the receiver would apply ideal filtering. The ACLR requirements are $>45 \mathrm{~dB}$ for a channels $>5 \mathrm{MHz}$ and 50 $\mathrm{dB}$ for $>10 \mathrm{MHz}$ [3]. In this article we only consider ACS requirements.

Moore's law is an important driver for the digitalization of the transceiver. The reason for this is that the digital part scales down with every new IC process generation and the analog part not. In addition, this digitalization allows the implementation of flexible radio architectures i.e. softwaredefined radio [4]. For manufacturers this could result in shorter development time, cheaper production due to higher volumes. Furthermore SDR has advantages for consumers because it enables only software updates for new functionality without new hardware.

In this paper we assume a SDR receiver architecture that holds the middle between a traditional and a highly digitized receiver architecture. To relax the processing power requirements of the digital part, the sampling frequency is chosen relatively low: $15.36 \mathrm{MHz}$ (i.e. four times the chip rate). The analog channel-selection filter is mainly used for image rejection. A more detailed description can be found in [5]. The analog channel-selection filter attenuates interfering signals at frequency offsets of $10 \mathrm{MHz}$ and higher by approximately $33 \mathrm{~dB}$. So the remaining $33 \mathrm{~dB}$ of attenuation at frequency offsets of $5 \mathrm{MHz}$ and higher will have to be provided by the digital channel-selection filter. The required stop-band attenuation can be realized by a 49-tap FIR filter.

\subsection{An adaptive digital filter}

In [6] Veljanovski et al. an adaptive digital receive filter has been described for a UMTS TDD (Time Division Duplex) handset receiver. We use this filter architecture as a starting point for the FDD case and a modified version is shown in Figure 1. It consists of a folded RRC (Root Raised Cosine) FIR (Finite Impulse Response) filter structure with an adjustable number of taps and a low-pass and a high-pass output, two rectifiers, two IIR (Infinite Impulse Response) lowpass filters and control logic. The rectified time-averaged output of the low-pass filter is a measure of the power inside of the desired UMTS channel and the output of the high-pass filter ${ }^{1}$ provides an estimate of the power outside the desired channel. Based on these outputs, the controller determines the required attenuation and the corresponding filter. When the attenuation and thus the number of used filter coefficients can be reduced, the required number of operations per filtered sample reduces as well, because less multiplications and additions have to be performed.

\footnotetext{
${ }^{1}$ The high-pass is easily constructed by subtracting the low-pass signal from the original signal (middle tap).
}

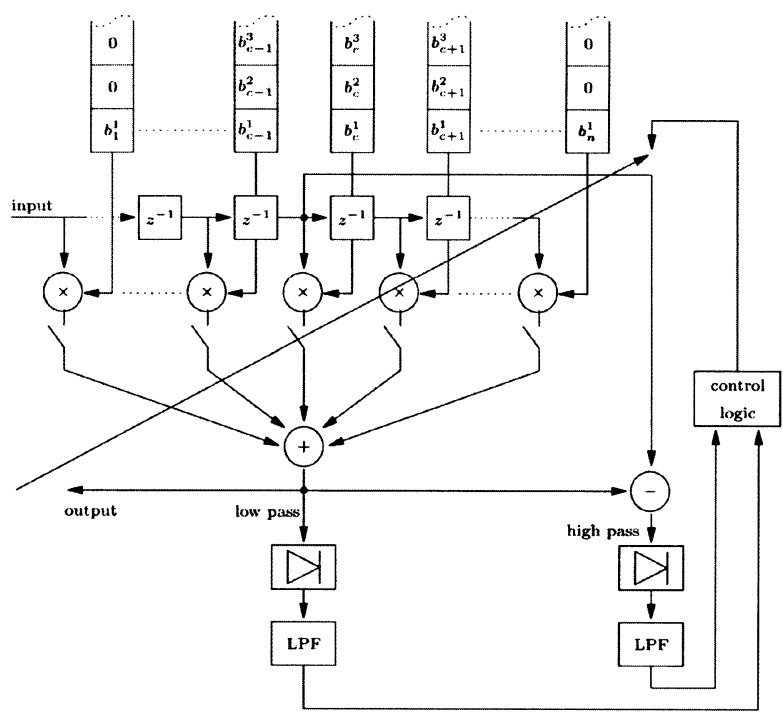

Fig. 1. Adaptive digital receive filter

\subsection{Filter coefficients}

In [7] Sevillano et al. an algorithm is proposed to design RRC FIR receive filters that trade off ICI (Inter Chip Interference) and stop-band attenuation. We have used this method to find the coefficients for a set of minimum length receive filters that achieve a desired range of stop-band attenuations, while the peak distortion remains below a desired threshold. According to [1], the EVM (Error Vector Magnitude) should be approximately $5 \%$ at maximum in order not to influence the performance of the receiver negatively. This means that the peak distortion should be $-20 \mathrm{~dB}$ or less.

In Figure 2, the results of this procedure and requirements for a range of desired attenuations from 6 to $34 \mathrm{~dB}$ with a step size of $1 \mathrm{~dB}$ is depicted. The dashed line with the cross markers indicates the number of used coefficients / attenuation combinations that can be achieved and the red line shows only valid points. The corresponding EVM and peak distortion are depicted in Figure 3 and Figure 4. The range of filter coefficient is between 9 and 37. So, if there is little interference in adjacent channels, a filter with 9 coefficients can be used. This means that the computation load of the receive filter can be largely reduced.

\subsection{Controller}

The optimized sets of receive filter coefficients can be used in the structure of Figure 1, in which not only the used number of coefficients is adjusted, but in which also the coefficients itself are changed. The controller works in a similar way as described in [6]. The rectified output of the low-pass filter is a measure for the signal level of the wanted channel and the rectified output of the high-pass filter is an indication for the 


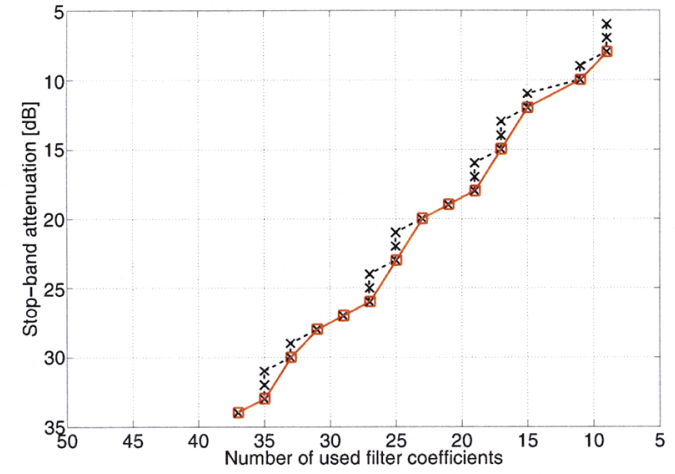

Fig. 2. Stop-band attenuation of optimized digital channelselection filters as a function of the number of used filter coefficients. (The dashed line with the cross markers indicates all combinations, the red line only depicts valid points.)

power of the out-of-band interference. Both values are used to estimate the required attenuation which is used to select the appropriate filter. The overhead of this extra hardware compared to a normal FIR filter is minimal: it has about the same complexity as eight taps of a FIR filter [8]. The overhead can be reduced even more if sub sampling is applied as the coherence time of the channel is typically several milliseconds. More research is required to verify this. In the next section, the results of an UMTS downlink measurement campaign are presented.

\section{UMTS DOWNLINK MEASUREMENTS}

In 2000, UMTS frequencies were awarded to 5 operators in the Netherlands in the $1.9 / 2.1 \mathrm{GHz}$ band. Although both FDD and TDD spectrum are available to operators, only FDD networks have been rolled out so far. For that reason the focus of this paper is on UMTS FDD. In 2006, we measured the field strength in the UMTS downlink band [8] at 47 locations in Amsterdam. Amsterdam is a dense urban region where all UMTS networks have been rolled out and active during our measurement campaign. At each location, the UMTS downlink band was measured at several points. See Figure 5 for the results of an example measurement.

In Table 1, the UMTS downlink spectrum assignment in the Netherlands is listed. Comparing Figure 5 with Table 1 clearly shows that each operator currently uses only one of their assigned channels at that particular location. This turns out to be the case for all 47 locations. Furthermore, the operators have chosen their active channels in such a way that there is at least one empty channel between their own active channel and the active channels of their competitors. So, currently a handset connected to any of the operators networks will not experience any interference for adjacent channel (ACI). Inter-

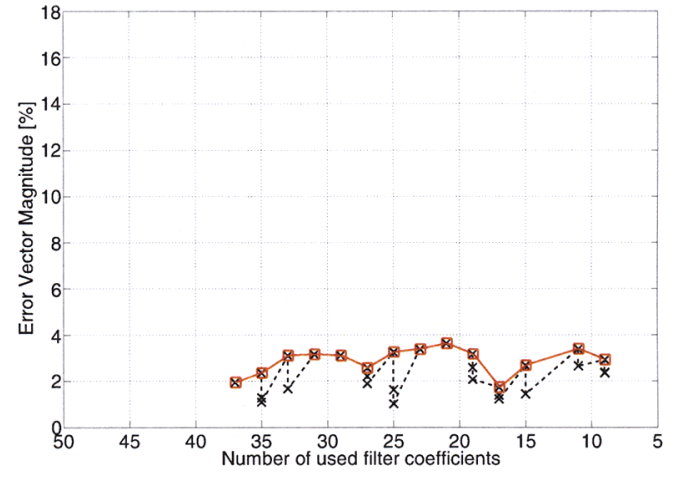

Fig. 3. EVM of optimized digital channel-selection filters as a function of the number of used filter coefficients. (The dashed line with the cross markers indicates all combinations, the red line only depicts valid points.)

ference from non-adjacent channels is less important as the analog front-end does part of the filtering (Section 2.1). For an operator this configuration also enables a fast network deployment as no coordination for base stations is required with competitors. ACI is also reduced in live network configurations, because often site-sharing occurs between operators.

\subsection{Signal statistics}

In total 376 measurements are used to determine the statistics of the signal conditions in the UMTS downlink band. From each measurement, the average field strengths in each of the UMTS channels are determined and used to determine the out-of-channel interference levels at offsets of \pm 2 or \pm 3 channels from a desired channel. The histograms of these measurements are shown in see Figure 6. An offset of \pm 1 is not shown as there is always an empty band of at least $5 \mathrm{MHz}$ ( 1 channel) between operators.

\subsection{Adjacent channel interference}

The maximum stop-band attenuation of the adaptive filter (33 $\mathrm{dB}$ ) is required (Section 2.1) if the measured $\mathrm{ACI}$ is $41 \mathrm{~dB}$ [8]. As the adjacent channel appears to be always empty in the Netherlands, no ACI will occur. This means that the on-demand receive filter can always select the filter with the minimal number of coefficients. The interference levels at larger offsets of \pm 2 and \pm 3 channels are higher than $41 \mathrm{~dB}$ for $0.50 \%$, respectively $0.27 \%$ of the measurements. So, if these networks were adjacent to the wanted channel, the maximum selectivity of the receive filter is hardly needed either.

The on-demand receive filter presented in this paper will become more computationally efficient compared to a fixed receive filter if the required stop-band attenuation is $10 \mathrm{~dB}$ 
Table 1. UMTS downlink spectrum assignment in the Netherlands.

\begin{tabular}{|c|c|c|c|c|c|c|c|c|c|c|c|c|}
\hline Operator & \multicolumn{3}{|c|}{ Vodafone } & \multicolumn{3}{|c|}{ KPN } & \multicolumn{2}{|c|}{ Orange } & \multicolumn{2}{|c|}{ Telfort } & \multicolumn{2}{|c|}{ T-Mobile } \\
\hline Freq. band [MHz] & \multicolumn{3}{|c|}{$2110.3-2124.9$} & \multicolumn{3}{|c|}{$2124.9-2139.7$} & \multicolumn{2}{|c|}{$2139.7-2149.7$} & \multicolumn{2}{|c|}{$2149.7-2159.7$} & \multicolumn{2}{|c|}{$2159.7-2169.7$} \\
\hline Channel [5 MHz BW] & 1 & 2 & 3 & 4 & 5 & 6 & 7 & 8 & 9 & 10 & 11 & 12 \\
\hline
\end{tabular}

Out of channel interference at offset of $+/-2$ channel(s)

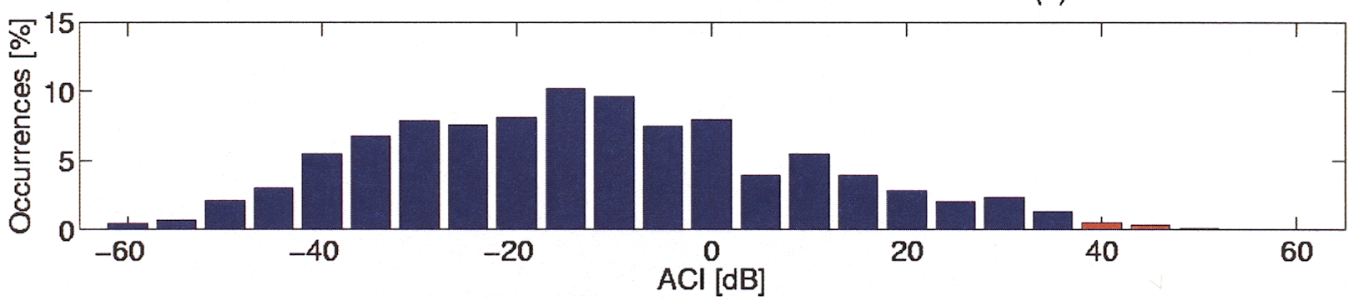

Out of channel interference at offset of $+/-3$ channel(s)

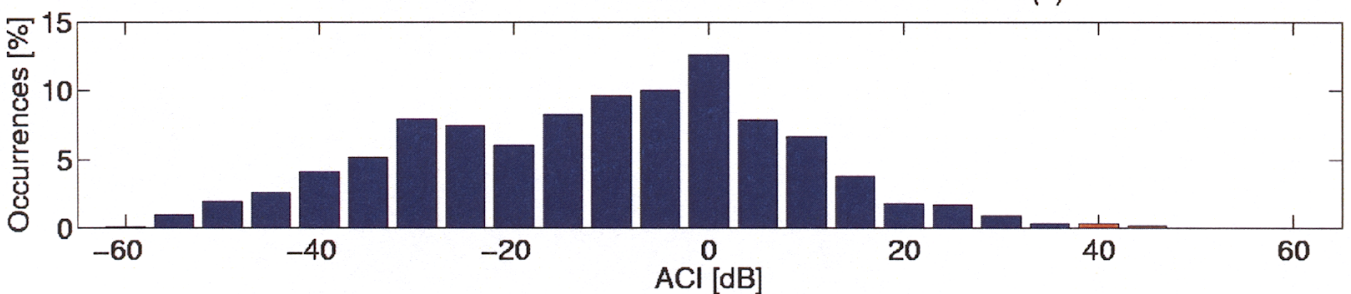

Fig. 6. Histograms of the measured out-of-channel interference levels in Amsterdam at offsets of \pm 2 or \pm 3 channels from the active channels. Orange is the range of ACI levels, where the necessary stop-band attenuation exceeds the requirements (of 33 $\mathrm{dB}$ ) for adjacent signals at a channel distance of $5 \mathrm{MHz}$.

less than the maximum required attenuation to compensate for the controller overhead. In this case, 8 taps can be shaved of the FIR filter, which is similar to the overhead of the controller. At offsets of \pm 2 and \pm 3 channels, this is the case for $97 \%$, respectively $99 \%$ of the measurements. So, an ondemand receive filter will be computationally more efficient than an UMTS handset with a fixed receive filter at least $97 \%$ of the time.

As GSM services are still more used than UMTS services in the Netherlands, it is reasonable to assume that the loads of the UMTS networks are currently relatively low. In addition, with the introduction and upgrade of UMTS networks with HSDPA (High-Speed Downlink Packet Access) in the Netherlands, we expect that the load of the UMTS networks will grow. So, the ACI may increase and in addition UMTS operators are likely to use more of their allocated spectrum. For these reasons, the results of the out-of-channel interference measurements might be on the optimistic side and hence the power savings of the on-demand filter might be lower.

\subsection{Computational savings}

The power consumption of the receive filter depends mainly on the number of multiplications per second. The filter coefficients of the adaptive filter range from 9 to 37 and the overhead of the controller is comparable to the computational load of 8 filter coefficients. So, if there is no out-of-channel interference the power consumption will be $25 \%((9+8) / 37)$ compared to a fixed filter that is designed for worst case conditions. However, if the conditions of the received filter are worst case, than the adaptive filter needs $21 \%((8+37) / 37)$ more power than a fixed filter.

In the previous section, we have shown that most of the time, there is no significant out-of-channel interference. If the adaptive receive filter uses a minimum amount of taps, the stop-band attenuation will be $8 \mathrm{~dB}$ (Figure 2) (and hence it can suppress a measured ACI of $16 \mathrm{~dB}$ ). In a realistic network configuration, only $10 \%$ of the time, extra filtering is required (Figure 6). If we assume that maximum filtering is required in this $10 \%$, we can estimate the average power saving of the ondemand receive filter. This power saving (i.e. lower bound) is: $0.90 \cdot 0.25+0.10 \cdot 1.21 \approx 0.32$. So, more than $68 \%$ of the power can be saved if the on-demand filter is applied in a UMTS handset. Of course this filter requires extra chip area, but due to Moore's law, extra costs are minimal.

\section{CONCLUSIONS}

In this paper, a novel on-demand receive filter in a UMTS terminal is presented. The receive filter is one of the computational intensive parts in a receiver and therefore significant 


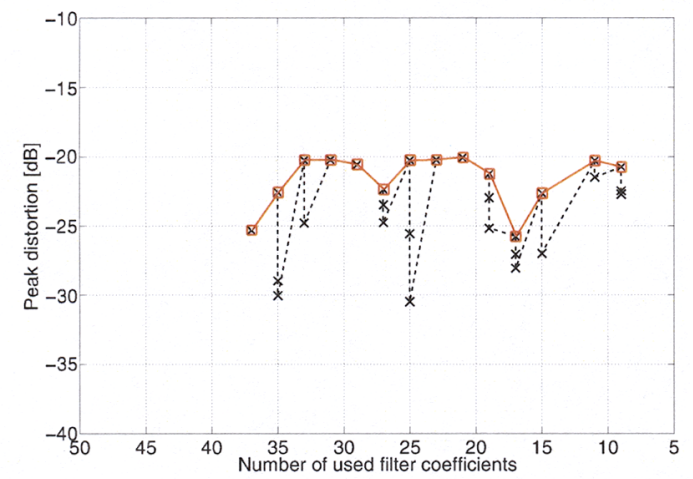

Fig. 4. Peak distortion of optimized digital channel-selection filters as a function of the number of used filter coefficients. (The dashed line with the cross markers indicates all combinations, the red line only depicts valid points.)

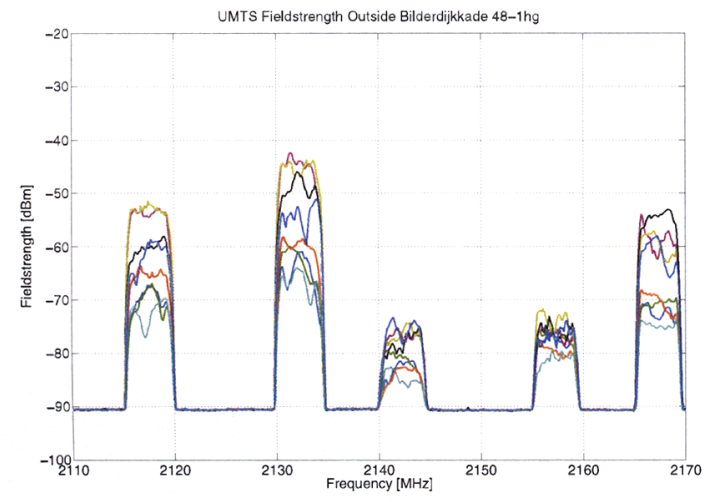

Fig. 5. Plot of an example field strength measurement (each line is a separate measurement)

power savings can be made if the filter is replaced by an ondemand filter. The proposed filter measures the out-of-band interference and calculates the required attenuation, which is used to select the appropriate filter

In Amsterdam, we have carried out field strength measurements in the UMTS FDD downlink band $(2.1 \mathrm{GHz})$ to assess the advantages of such an adaptive filter in a live network configuration. (In the Netherlands 5 UMTS networks were active during the measurement campaign.) We measured in $90 \%$ of the time no out-of-band interference. As a result, more than $68 \%$ power can be saved compared to a fixed receive filter. Further research focusses on reducing the overhead of the on-demand filter compared to a fixed filter. Reducing the overhead will increase power savings even more.

\section{REFERENCES}

[1] J. Jussila, Analog Baseband Circuits for WCDMA DirectConversion Receivers, Ph.D. thesis, Helsinki University of Technology, 2003.

[2] Third Generation Partnership Project, "Universal Mobile Telecommunications System (UMTS); UE Radio transmission and reception (FDD)," Tech. Rep. ETSI TS 125 101 V8.2.0, European Telecommunications Standards Institute, April 2008.

[3] Third Generation Partnership Project, "Universal Mobile Telecommunications System (UMTS); UE Radio transmission and reception (FDD)," Tech. Rep. ETSI TS 125 104 V8.2.0, European Telecommunications Standards Institute, April 2008.

[4] J. Mitola, Software radio architecture, John Wiley \& Sons,Inc., 2000.

[5] J. Potman, F. W. Hoeksema, and C. H. Slump, "Adaptive receive filter structures for UMTS," in Proceedings of SPS-DARTS 2006, the second annual IEEE Benelux/DSP Valley Signal Processing Symposium, Antwerp, Belgium, Antwerp, March 2006, pp. 55-59, IEEE Benelux EMBS.

[6] R. Veljanovski, J. Singh, and M. Faulkner, "A Proposed Reconfigurable Digital Filter for a Mobile Station Receiver," in Proceedings of GLOBECOM '02, November 2002, pp. 524-528.

[7] J.F. Sevillano, I. Vélez, and A. Irizar, "On the Design of Receiver Root-Raised Cosine FIR Filters," IEEE Transactions on Consumer Electronics, vol. 51, no. 4, pp. 1104-1109, November 2005.

[8] J. Potman, F. W. Hoeksema, and C. H. Slump, "Adjacent channel interference in UMTS networks," in Proceedings of ProRISC the 17th Annual Workshop on Circuits, Systems and Signal Processing, Veldhoven, The Netherlands, Veldhoven, The Netherlands, November 2006, pp. 165-170, STW. 